\title{
Inducing Macrophages M2 Polarization by Dexamethasone Laden Mesoporous Silica Nanoparticles from Titanium Implant Surface for Enhanced Osteogenesis
}

\author{
Jing Luo ${ }^{1,2} \cdot$ Xin Ding ${ }^{3} \cdot$ Wen Song ${ }^{1} \cdot$ Jian-Ying Bai ${ }^{1} \cdot$ Jing Liu $^{1} \cdot \mathrm{Zhe} \mathrm{Li}^{1} \cdot$ Fan-Hui Meng ${ }^{4} \cdot$ Fang-Hao Chen ${ }^{1}$. \\ Yu-Mei Zhang ${ }^{1}$
}

Received: 31 March 2019 / Revised: 25 April 2019 / Published online: 18 June 2019

(c) The Chinese Society for Metals (CSM) and Springer-Verlag GmbH Germany, part of Springer Nature 2019

\begin{abstract}
The study conveys an idea to enhance the osseointegration of titanium implant (Ti) through modulating macrophages M2 polarization. The $\sim 100 \mathrm{~nm}$ spherical mesoporous silica nanoparticles (MSN) that compromised of $\sim 4$-nm-diameter nanotunnels were synthesized by the conventional "sol-gel" method, into which the dexamethasone (DEX) was loaded (DEX@ MSN). The DEX@MSN could consistently release DEX and showed favorable cytocompatibility in RAW264.7 cells. The arginase-1 expression, a specific marker for macrophages M2 polarization, was also enhanced by DEX@MSN treatment. Then, the Ti was pre-treated with anodization under $5 \mathrm{~V}$ to generate the titania nanotubes with $\sim 30 \mathrm{~nm}$ diameter (NT-30) and the DEX@MSN was introduced onto NT-30 surface via electrophoretic deposition, with the aid of chitosan. After optimizing the deposition parameters, the supernatants of RAW264.7 from the decorated implant surface could significantly promote the osteogenic differentiation of murine primary bone marrow mesenchymal stem cells. These findings demonstrate that delivery of DEX from implant surface can modulate the macrophages M2 polarization and result in favorable osteogenesis.
\end{abstract}

Keywords Macrophages polarization · Titanium implant · Osteogenesis $\cdot$ Dexamethasone $\cdot$ Mesoporous silica nanoparticles $\cdot$ Electrophoretic deposition

\section{Introduction}

Available online at http://link.springer.com/journal/40195

Wen Song

songwenfmmu@hotmail.com

Yu-Mei Zhang

wqtzym@fmmu.edu.cn

1 State Key Laboratory of Military Stomatology and National Clinical Research Center for Oral Diseases and Shaanxi Key Laboratory of Oral Diseases, Department of Prosthodontics, School of Stomatology, The Fourth Military Medical University, Xi' an 710032, China

2 Department of Stomatology, General Hospital of Southern Theater Command of the Chinese People's Liberation Army, Guangzhou 510010, China

3 Huaian Stomatological Hospital, Huaian 223001, China

4 State Key Laboratory of Military Stomatology and National Clinical Research Center for Oral Diseases and Shaanxi Key Laboratory of Oral Diseases, Department of Dental Materials, School of Stomatology, The Fourth Military Medical University, Xi' an 710032, China
The role of macrophages polarization in tissue remodeling has attracted more and more attention [1,2]. It is reported that the polarization directions of macrophages would result in distinct cytokines secretion and thus influence the microenvironment of titanium (Ti) implant [3]. Specifically, the cytokines secreted by M2 polarized macrophages are beneficial for new bone formation, whereas the M1-polarized macrophages are attributed to the inflammatory responses [4]. Therefore, harnessing the macrophages polarization toward M2 direction has become a new strategy in tissue engineering [5].

Inducing macrophages M2 polarization has become an attractive strategy to improve bone formation through modulating the microenvironment [6]. There are numerous ways to induce macrophages $\mathrm{M} 2$ polarization, among which the IL-4/IL-13 stimulus is considered as a standard procedure [7]. Indeed there are reports to suggest that delivery of IL-4/ IL-13 from implant surface is able to trigger macrophages M2 polarization and the consequent tissue favoring [8]. 
However, the cytokines are of high cost and are vulnerably denatured. Dexamethasone (DEX) is generally used for inflammation inhibition in various situations such as severe infection, autoimmune diseases and allergy [9]. In the meanwhile, DEX is well known as a powerful drug to induce M2 polarization in human blood-derived monocytes [10]. Moreover, it is also an essential component in the osteogenic medium for bone marrow mesenchymal stem cells (BMSCs) differentiation in vitro [11]. However, the side effects of DEX after long-term intake systematically are also unacceptable [9]. As a result, local and controlled delivery of DEX from implant surface may work more efficient and safer. Decoration of titanium implant surface with DEX may acquire significant enhancement of osteogenic differentiation of BMSCs.

In the present study, the mesoporous silica nanoparticles (MSN) were synthesized to adsorb DEX (denoted as DEX@MSN), which were immobilized onto the Ti implant surface for enhancing the osteogenesis through modulating macrophages M2 polarization (Fig. 1).

\section{Materials and Methods}

\subsection{MSN and DEX@MSN Synthesis and Observation}

MSN was prepared by a "sol-gel" method of mixing tetraethyl silicate orthosilicate (TEOS) with the template surfactant cetyltrimethylammonium bromide (CTAB) in an aqueous alkaline solution ( $\mathrm{pH} \mathrm{11),} \mathrm{as} \mathrm{described} \mathrm{in} \mathrm{the} \mathrm{lit-}$ erature [12]. For DEX@MSN preparation, 0.2 g MSN was suspended into $20 \mathrm{ml}$ dexamethasone solution $(0.1 \mathrm{mg} / \mathrm{ml}$ in absolute ethanol) and stirred for $24 \mathrm{~h}$ at room temperature [13]. The DEX@MSN was collected by centrifugation, washed three times with phosphate buffer saline (PBS), and dried in vacuum.

The morphology and structure of nanoparticles were observed by using a transmission electron microscope (TEM, FEI Tecnai Spirit, USA), scanning electron microscope (SEM, S-4800, Hitachi, Japan) and atomic force microscope (AFM, Dimension Icon, Bruker, Germany), respectively.

\subsection{Loading and Release Profiles of DEX@MSN}

For DEX quantification, the calibration curve was prepared by measuring the optical densities of various concentrations of DEX solutions at $242 \mathrm{~nm}$ according to other report [13]. To determine the maximum DEX loading capacity, excess DEX was initially used for MSN adsorption and the loaded DEX was calculated as the difference between initial DEX and residuals in the supernatants from each centrifugation step.

The release profile of DEX from DEX@MSN particles was determined as reported elsewhere [13]. The DEX@ MSN was dispersed in PBS and stored in a dialysis bag (3500 Da). After sealing, the dialysis bag was immersed in PBS and incubated at $37^{\circ} \mathrm{C}$ with orbital shaking. The dialysate was taken at different time points to determine the released DEX amount, and the cumulative release was depicted as the percentage to total adsorbed DEX.

\subsection{Cell Culture}

Murine macrophage cell line RAW264.7 (ATCC, USA) was cultured in Dulbecco's modified Eagle medium (DMEM) (Carlsbad, CA) containing 10\% fetal bovine serum (FBS), $1 \%$ penicillin/streptomycin. The cells were passaged at

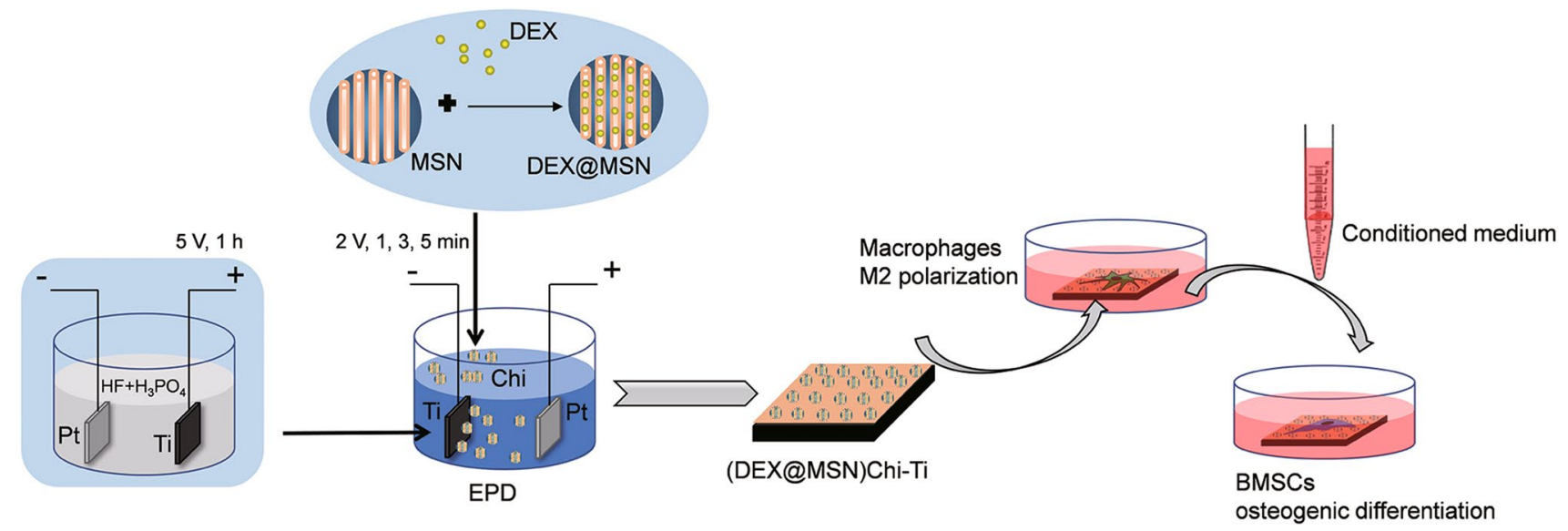

Fig. 1 Illustration of experimental design. The DEX was loaded into MSN to formulate the DEX@MSN particles, which were immobilized onto pre-anodized Ti implant surface through EPD. The functionalized Ti implant was able to induce macrophages M2 polarization, which in turn contributed to BMSCs osteogenic differentiation 
$70-80 \%$ confluence every $2-3$ days. The murine BMSCs were isolated according to the previous reports [14], under the approval of the University Research Ethic Committee of The Fourth Military Medical University.

\subsection{CCK-8 Assay}

The RAW264.7 was seeded at a density of $5 \times 10^{4}$ cells/ well in 96-well plates. After $24 \mathrm{~h}$, various concentrations of MSN or DEX@MSN nanoparticles were added. After incubation for another 1 or 3 days, the cell viability was evaluated by CCK- 8 assay (Beyotime, China) according to the manufacturer's instructions. Briefly, the cells were rinsed in PBS and treated with CCK- 8 work solution (dilution of the CCK-8 stock solution in full medium at the ratio of 1:10). After incubation at $37^{\circ} \mathrm{C}$ for $3 \mathrm{~h}$, the absorbance was read at $450 \mathrm{~nm}$ by a plate reader. The untreated cells were set as $100 \%$ to normalize the cell viability. For the evaluation of (DEX@MSN)Chi-Ti surface, the implant was placed in a 24-well plate and the RAW264.7 cells were seeded at a density of $5 \times 10^{5}$ cells/well. After 1 and 3 days, CCK-8 assay was carried out similarly.

\section{5 qPCR Analysis}

RAW264.7 cells were seeded into 24-well plates at a density of $5 \times 105$ cells/well and received treatments of DEX@MSN or free DEX for 3 days. The M2 polarization-specific marker arginase $1(\operatorname{Arg} 1)$ was measured by real-time quantitative polymerase chain reaction (qPCR) analysis [15]. Briefly, the total RNA was extracted by RNAiso Plus (Takara, Tokyo, Japan) according to the manufacturer's instructions, which was used as the template for cDNA synthesis by using PrimeScriptTM RT Master Mix (Perfects Real Time; Takara). The qPCR was performed by SYBR ${ }^{\circledR}$ Premix Ex TaqTMII on a CFX96 system (Bio-Rad), with the Arg 1 primer sequence of Forward 5'-AGCTCTGGGAATCTGCATGG-3' and Reverse 5'-ATGTACACGATGTCTTTGGCAGATA-3' [16]. The glyceraldehyde-3-phosphate dehydrogenase (GAPDH) was used as housekeeper gene, with the primer sequence of Forward 5'-CCAATGTGTCCGTCGTGGATCT-3' and Reverse 5'-GTTGAAGTCGCAGGAGACAACC-3' [17]. For the evaluation of DEX@MSN-decorated Ti implant surface, the implant was placed in a 24-well plate and incubated with RAW264.7 cells at a density of $5 \times 10^{5}$ cells/well for 3 days. The Arg1 mRNA level was measured by similar qPCR analysis.

\subsection{Preparation of (DEX@MSN)Chi-Ti}

Pure Ti square plates (side length $=10 \mathrm{~mm}$, thickness $=1 \mathrm{~mm}$, Northwest Institute for Nonferrous Metal Research, $\mathrm{Xi}$ ' an, China) were polished by $\mathrm{SiC}$ sandpapers from $800 \#$ to $7000 \#$. The Ti implant surface with $\sim 30 \mathrm{~nm}$ titania nanotubes (NT-30) was fabricated according to our previous report [14]. The (DEX@MSN)Chi-Ti was fabricated by electrophoretic deposition (EPD) process according to the related report [18]. Briefly, the chitosan $(\mathrm{Mw}=100-300 \mathrm{kD}, \mathrm{DD}=85-95 \%$, Sigma-Aldrich Company, USA) was dissolved in $2 \%(\mathrm{v} / \mathrm{v})$ acetic acid at the concentration of $1 \mathrm{mg} / \mathrm{ml}$, and the same volume of absolute ethanol was added dropwise under continuous magnetic stirring. The DEX@MSN (100 mg)was ultrasonically dispersed in the aforementioned chitosan solution $(200 \mathrm{ml})$ to serve as the electrolyte, while the Ti implant and platinum were set as the cathode and anode, respectively. The EPD process was carried out under $2 \mathrm{~V}$ for 1,3 , and $5 \mathrm{~min}$, respectively, at room temperature, and the fabricated (DEX@MSN)Chi-Ti samples were dried in vacuum prior to the SEM examination.

\subsection{Alizarin Red Staining}

The RAW264.7 cells were cultured on (DEX@ MSN)Chi-Ti surface for 3 days, and the supernatants were collected. The conventional osteogenic medium was prepared by supplementing ascorbic acid $(50 \mu \mathrm{g} / \mathrm{ml})$, dexamethasone $(10 \mathrm{nM})$ and $\beta$-glycerophosphate $(10 \mathrm{mM})$ into normal BMSCs culturing medium. The conditioned medium was prepared by mixing the macrophages supernatants and conventional osteogenic medium at the ratio of 1:1 [9]. The BMSCs were cultured on (DEX@MSN)Chi-Ti surface to perform the osteogenic differentiation in conditioned medium for 14 days. Then, the cells were fixed in $4 \%$ paraformaldehyde for $30 \mathrm{~min}$ at room temperature and stained by Alizarin Red ( $\mathrm{pH}=4.2$, Sigma, USA) for $30 \mathrm{~min}$. After washing thoroughly with PBS, the samples were imaged by a stereomicroscope (Leica, Germany). Afterward, the stains were dissolved by $10 \%$ cetylpyridinium chloride, and the absorbance was measured by a plate reader at $620 \mathrm{~nm}$.

\subsection{ALP Activity}

The BMSCs were cultured on (DEX@MSN)Chi-Ti surface to perform the osteogenic differentiation in conditioned medium for 7 days. Then, the intracellular ALP activity was determined by BCIP/NBT ALP Color Development Kit (Beyotime, China) and ALP quantification assay kit (Nanjing Jiancheng Bioengineering Institute, China), respectively, according to the manufacturer's instructions. Briefly, for the staining kit, the cells were fixed in $4 \%$ paraformaldehyde for $30 \mathrm{~min}$ at room temperature and rinsed by PBS for three times. Then, the cells were treated with BCIP/NBT work solution for $30 \mathrm{~min}$ at room temperature, protected from light. The staining was terminated by rinsing the cells with deionized water, and the images were taken immediately by stereomicroscope (Leica, Germany). For the quantification 
assay, the cells were lysed by freeze-thaw cycles in $1 \%$ Triton X-100. Then, the cell lysates were centrifuged for $15 \mathrm{~min}$ at $4{ }^{\circ} \mathrm{C}$ and the supernatants were collected. After reactions with substrate for $15 \mathrm{~min}$ at $37^{\circ} \mathrm{C}$, the optical densities were measured at $405 \mathrm{~nm}$ to determine the relative ALP activity, which was normalized by intracellular total protein that measured by the BCA Protein Assay Kit (Beyotime, China).

\subsection{Statistical Analysis}

All experiments were performed at least three times, and the quantitative data were expressed as mean \pm standard deviation. The comparison of different means was evaluated by one-way ANOVA, in company with Dunnett's test and $P<0.05$ was set as statistical significance.

\section{Results}

\subsection{MSN and DEX@MSN Characterizations}

The MSN exhibited characteristic channels in the lateral view and hexagonal pores in the frontal view, and the particle size could be estimated as $\sim 100 \mathrm{~nm}$ while the channels diameter was $\sim 4 \mathrm{~nm}$ (Fig. 2a-c). The MSN surface topography was scanned by AFM. From bottom to the top, the height increased continuously without gaps, suggesting that there was no defect on particle surface (Fig. 2d).
After adsorption of DEX, the particle size did not change apparently, whereas the channels and pores structures were invisible under TEM observation (Fig. 2e). The SEM image also showed that the particle surface was smooth without apparent pores (Fig. 2f). The calibration curve of DEX concentration to the optical density at $242 \mathrm{~nm}$ showed a fine linear relationship (Fig. 2g). The maximum loading amount of DEX was about $45.5 \mu \mathrm{g} / \mathrm{mg}$. The DEX@MSN was incubated in PBS, and the released DEX was determined. Within the checked time points, the DEX was released steadily and slowly that less than $30 \%$ was released after 4 days incubation (Fig. 2h).

\subsection{Cytotoxicity and M2 Polarization Induction of DEX@MSN}

MSN showed dose-dependent cytotoxicity, and cell viability was significantly inhibited when the concentration was above $2 \mu \mathrm{g} / \mathrm{ml}$ (Fig. 3a). DEX @MSN also showed similar dose-dependent cytotoxicity, and cell viability was significantly inhibited when the concentration was above $5 \mu \mathrm{g} / \mathrm{ml}$ (Fig. 3b). qPCR was used to detect the mRNA expression level of the M2 polarization-specific marker Arg1. Low concentrations of DEX treatment significantly inhibited Arg1 expression (Fig. 3c). However, when the concentration was increased to $100 \mathrm{nM}$ and $1000 \mathrm{nM}$, Arg1 expression was enhanced to nearly 30 -fold compared to untreated cells (Fig. 3c). The DEX@ MSN treated cells also showed
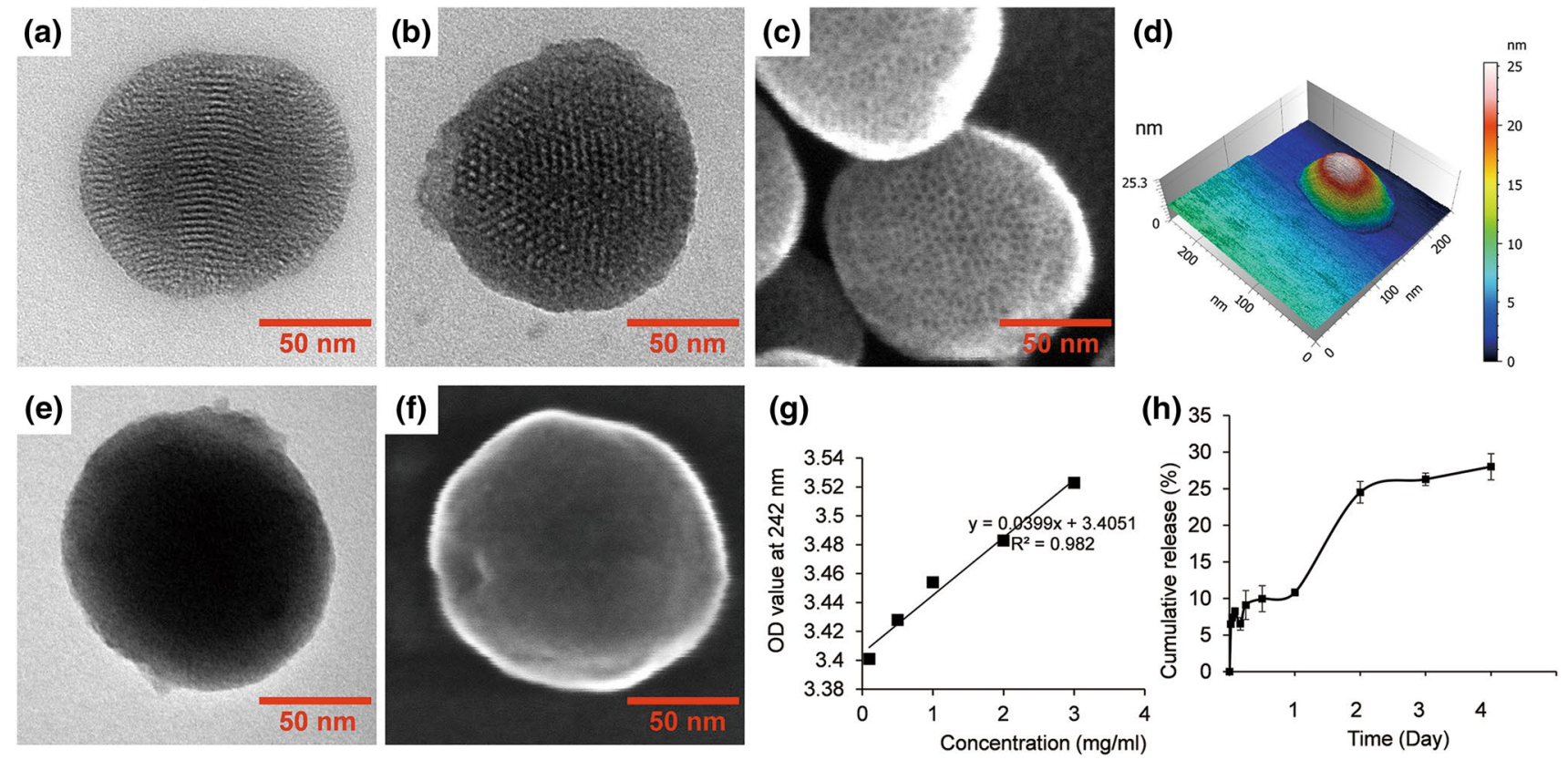

Fig. 2 Characterizations of MSN and DEX@MSN. a The lateral view of MSN under TEM. b The frontal view of MSN under TEM. c The SEM image of MSN. d The surface height image of MSN by AFM scanning. e TEM image of DEX@MSN. f The TEM image of DEX@MSN. $\mathbf{g}$ The linear relationship between optical density (OD) values and DEX concentrations. h The cumulative released DEX percentage from DEX@MSN particles 

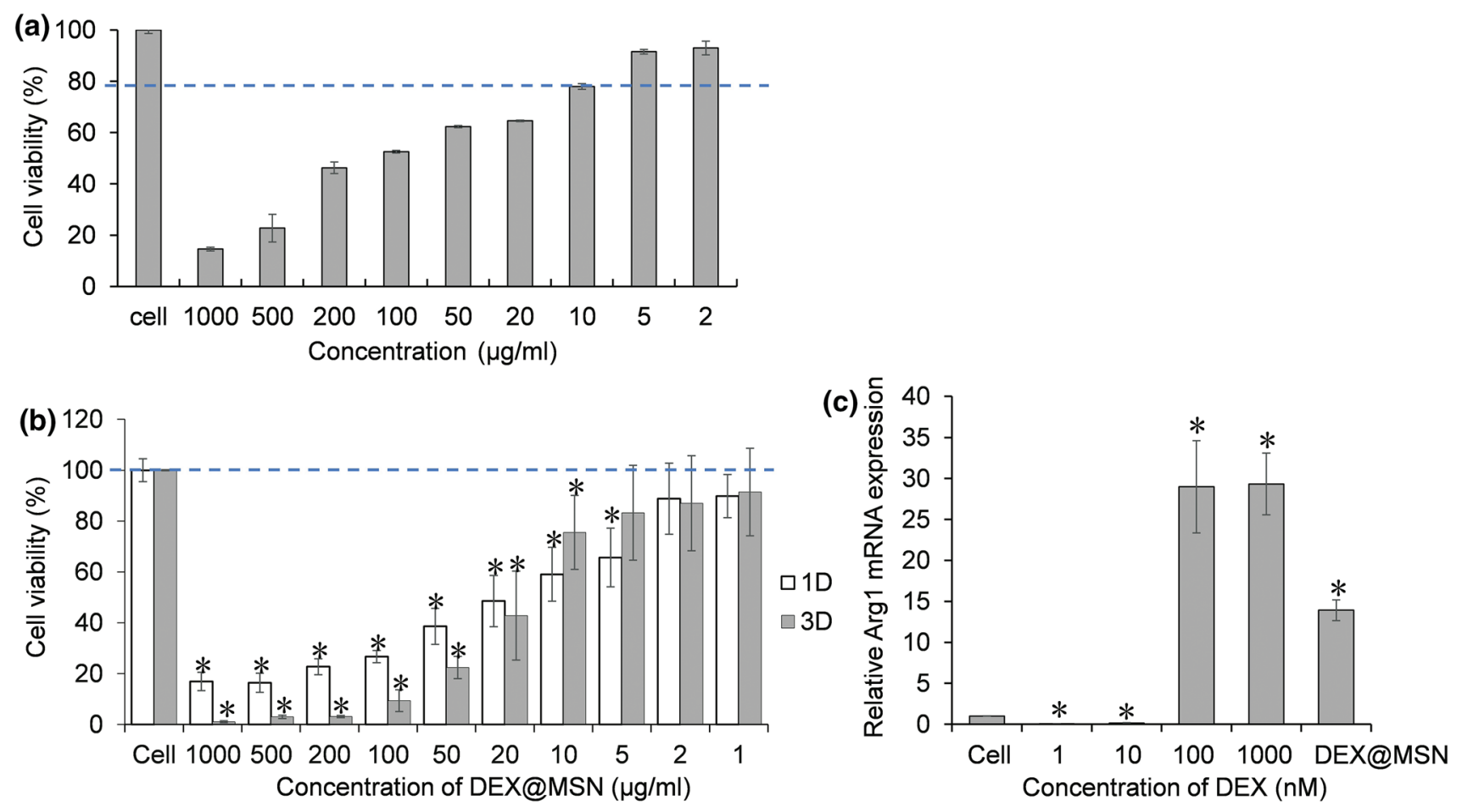

Fig. 3 Cytotoxicity and M2 polarization induction of DEX@MSN. The RAW264.7 cells were treated with various concentrations of MSN, and the cell viability was checked by CCK-8 assay after culture for 1 day a. The RAW264.7 cells were treated with various concentrations of DEX@ MSN, and the cell viability was checked by CCK-8 assay after culture for 1 and 3 days $\mathbf{b}$. The RAW264.7 cells were treated with various concentrations of DEX and $2 \mu \mathrm{g} / \mathrm{ml}$ DEX@MSN. Total intracellular RNA was isolated to perform the qPCR analysis of Arg1 c. ${ }^{*} P<0.05$ versus cell

a tenfold higher expression of Arg1 compared to untreated cells (Fig. 3c).

\section{3 (DEX@MSN)Chi-Ti Observation, Cytotoxicity and M2 Polarization Induction}

The surface morphology of deposited Ti samples was examined by SEM under various magnifications (Fig. 4a). At lower magnification, the NT-30 surface was occupied by conventional micro-scale etched pits. At higher magnification, uniform nanotubes with an average diameter of $\sim 30 \mathrm{~nm}$ were evenly aligned. The (DEX@MSN)Chi-Ti samples showed isolated patches under lower magnification and with the increase in deposition time, the patches area was expanded. By zooming in the patches, it was clear that the nanoparticle clusters were embedded in chitosan film. By further enlarging the nanoparticle clusters, the smooth characteristic DEX@MSN morphology was presented. The cytotoxicity was evaluated by CCK- 8 assay (Fig. 4b). The cell viability was suppressed on the naked NT-30 surface. Although slightly decreased, the cell viability on (DEX@ MSN)Chi-Ti was maintained more than $85 \%$. The Arg1 expression was determined by qPCR (Fig. 4c). The NT-30 surface showed inhibition on Arg1 expression. The Arg 1 expression was also not enhanced on (DEX@MSN)Chi-Ti after $1 \mathrm{~min}$ and 3 min deposition. However, after 5 min deposition, the (DEX@MSN)Chi-Ti surface significantly enhanced Arg1 expression to more than fivefold.

\subsection{Osteogenic Differentiation Under Conditioned Medium}

The osteogenic differentiation of BMSCs was performed in the presence of the related conditioned medium. The calcium nodules formation was significantly augmented by receiving the macrophages conditioned medium that originated from EPD surface (Fig. 5a). Similarly, the ALP activity was also enhanced $\sim$ threefold higher (Fig. $5 b$ ).

\section{Discussion}

Although the biomimetic approach shows great potential in the biomaterials designation [19], the microenvironment is a key factor for tissue remodeling, in which macrophages play the decisive role [6]. Accordingly, the secretion products of M2 polarized macrophages are beneficial for bone formation [20]. Therefore, it has become a prominent strategy to induce M2 polarization of macrophages, thus regulating osteogenic microenvironment to improve bone formation 

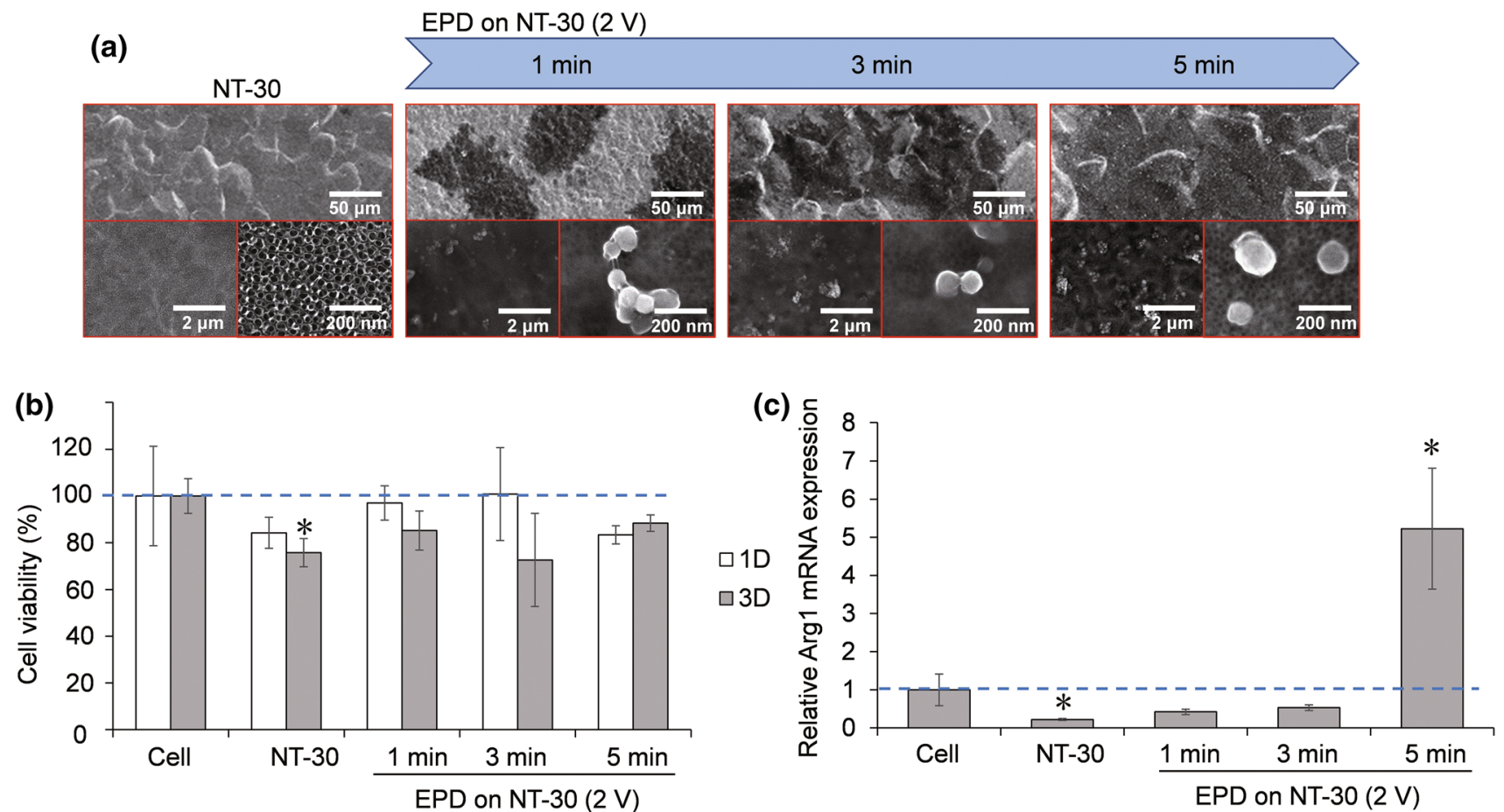

Fig. 4 (DEX@MSN)Chi-Ti observation, cytotoxicity and M2 polarization induction. a The Ti implant was anodized at $5 \mathrm{~V}$ to formulate the NT-30 surface. Then, the EPD was performed for different times to immobilize DEX@MSN. The samples were freeze-dried and examined by SEM. b The RAW264.7 cells were cultured on (DEX@MSN)Chi-Ti surface for 1 and 3 days. The cell viability was determined by CCK-8 assay. $* P<0.05$ versus cell. $\mathbf{c}$ The RAW264.7 cells were cultured on (DEX@MSN)Chi-Ti surface for 3 days. Total intracellular RNA was isolated to perform the qPCR analysis of Arg $1 . * P<0.05$ versus cell
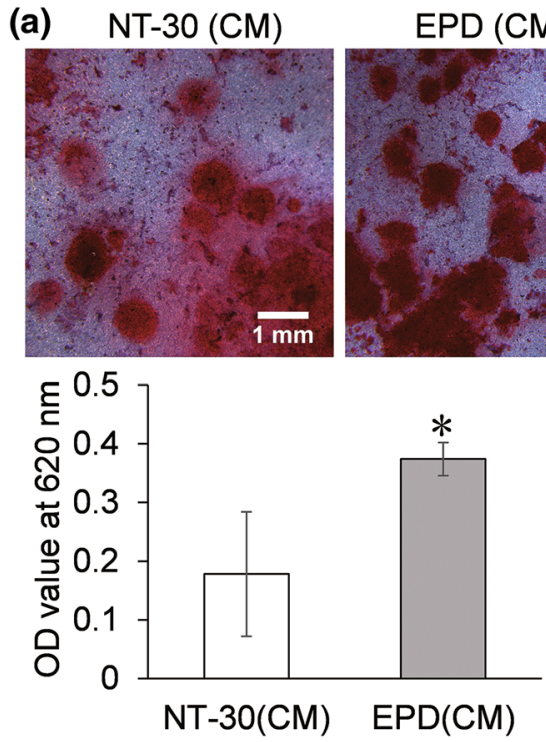

(b)
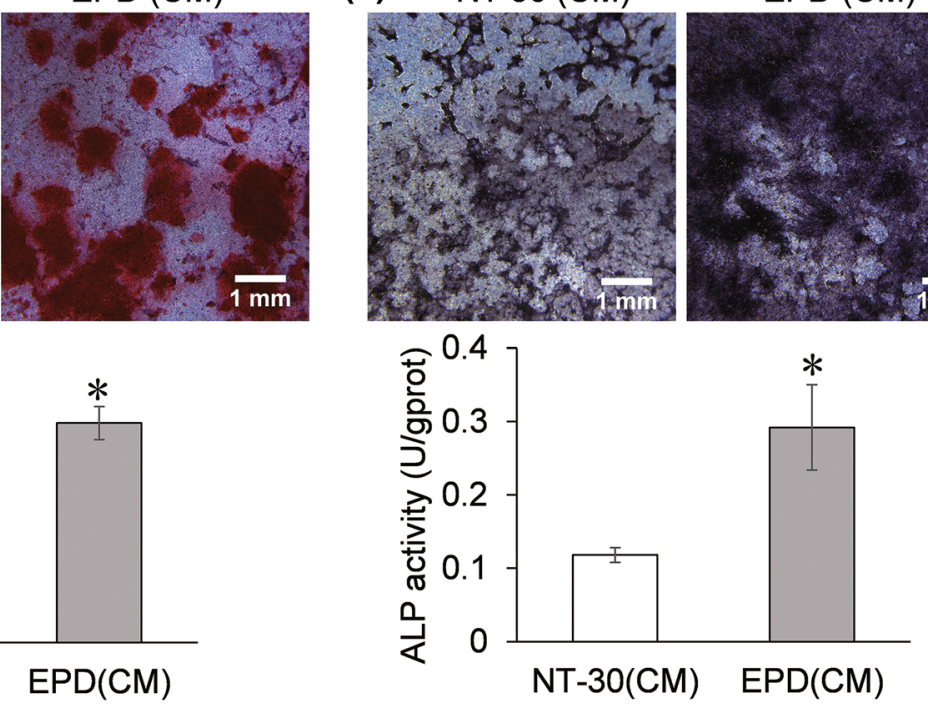

Fig. 5 Osteogenic differentiation of BMSCs after receiving macrophages conditioned medium. The RAW264.7 cells were cultured on different surfaces, and the supernatants were collected to mix with conventional osteogenic medium to formulate the conditioned medium. The BMSCs were cultured on NT-30 surface and subjected to osteogenic differentiation in conditioned medium. The Alizarin Red staining a and ALP activity $\mathbf{b}$ were performed to evaluate the osteogenic differentiation efficacy. $* P<0.05$

[6]. Titanium dioxide nanotubes (NT) have been well studied previously $[21,22]$. Our previous study demonstrated that the $30 \mathrm{~nm}$ diameter NT (NT-30) has an intrinsic potential to induce macrophage polarization toward the M2 direction 
[14]. However, the inductive effects of topography are very limited and it is difficult to efficiently meet clinical needs. Therefore, this study combines exogenous stimulating factors with the intrinsic induction of topography to work together.

The MSN has been extensively studied as a carrier for drug delivery due to its unique structures [23]. Accordingly, the MSN is a nanosized sphere, which is compromised by a bundle of nanoscale hexagonal channels [24]. This is consistent with our observations that a number of regular hexagonal channels build up to formulate a regular round particle, without apparent defects on the surface. After encapsulation of DEX, the channels and pore structures are invisible and the surface is also quite smooth, indicating that the drugs are successfully packed into MSN.

By the cell viability test, it is clear that the MSN does have an impact on cell viability, which is concentration dependent. Indeed, over dosage uptake of MSN may cause direct cell damage. Some suggest that the overdose uptake of MSN may trigger excess ROS generation and cause oxidative damage $[25,26]$. Others indicate that the MSN may damage the endosome/lysosome system and result in lysosomes dysfunction [27]. In addition, if the accumulation of MSN cannot be degraded in time, it may result in organelles disruption and induce cell apoptosis [28]. Anyway, by tuning the DEX@MSN in a proper concentration range, the cells are endurable and can significantly increase the Arg1 expression, a specific marker for M2 macrophages, indicating a strong induction for M2 polarization.

The EPD is widely used as a versatile method for noncovalent coating fabrication [29-31]. Under direct current, the charged nanoparticles can migrate to the counter electrodes in several minutes, which is both highly efficient and controllable. Unfortunately, the DEX@MSN is nearly neutral, which cannot be used for EPD directly. Therefore, the incorporation of chitosan coating to acquire the positive charge is used for EPD procedure, which is also developed for the siRNA immobilization in our previous study [32]. From the SEM observation, it is clear that the DEX@ MSN particles are embedded in chitosan films. By adjusting deposition parameters, the cytotoxicity of decorated implant surface can be controlled in a safe range, which is also a prerequisite for in vivo application. In addition, there is a significant increase in Arg1 mRNA expression after DEX@ MSN decoration at 5 min deposition, indicating that the system is able to induce macrophages M2 polarization.

In order to confirm the idea that the osteogenic microenvironment plays an important role for bone formation around implant, the indirect co-culture system has been deployed. As expected, the conditioned medium from (DEX@MSN) Chi-Ti surface has greatly enhanced the osteogenic differentiation of BMSCs. This has confirmed our idea that the osteogenic microenvironment is of crucial importance. It has not only provoked a new concept during biomaterials design, but also required us to re-consider the relationship between immune system and tissue regeneration.

\section{Conclusion}

Based on our previous findings of the determinant role of macrophages polarization in bone formation, the current study has established a localized DEX@MSN eluting titanium implant surface for improving osteogenic induction capacity. The MSN is able to store DEX for a long duration, which is beneficial for inducing sustained macrophages M2 polarization and enhanced osteogenic differentiation of BMSCs. However, the MSN shows dose-dependent cytotoxicity, which needs to be improved in future investigations.

Acknowledgements This work was supported by the National Natural Science Foundation of China (Nos. 31800790, 81530051 and 31670966).

\section{References}

[1] H. Kang, S. Kim, D. Wong, H.J. Jung, S. Lin, K. Zou, R. Li, G. Li, V.P. Dravid, L. Bian, Nano Lett. 17, 6415 (2017)

[2] P. Krzyszczyk, R. Schloss, A. Palmer, F. Berthiaume, Front. Phys. 9, 419 (2018)

[3] J. Wang, S. Qian, X. Liu, L. Xu, X. Miao, Z. Xu, L. Cao, H. Wang, X. Jiang, J. Mater. Chem. B 5, 3364 (2017)

[4] D.M. Mosser, J.P. Edwards, Nat. Rev. Immunol. 8, 958 (2008)

[5] K.L. Spiller, S. Nassiri, C.E. Witherel, R.R. Anfang, J. Ng, K.R. Nakazawa, T. Yu, G. Vunjak-Novakovic, Biomaterials 37, 194 (2015)

[6] R. Sridharan, A.R. Cameron, D.J. Kelly, C.J. Kearney, F.J.O. Brien, Mater. Today 18, 313 (2015)

[7] B. Cha, S.R. Shin, J. Leijten, Y. Li, S. Singh, J.C. Liu, N. Annabi, R. Abdi, M.R. Dokmeci, N.E. Vrana, A.M. Ghaemmaghami, A. Khademhosseini, Adv. Healthc. Mater. 6, 1700289 (2017)

[8] D. Hachim, S.T. LoPresti, C.C. Yates, B.N. Brown, Biomaterials 112, 95 (2017)

[9] P. Li, Y. Zheng, X. Chen, Front. Pharmacol. 8, 460 (2017)

[10] S. Tedesco, C. Bolego, A. Toniolo, A. Nassi, G.P. Fadini, M. Locati, A. Cignarella, Immunobiology 220, 545 (2015)

[11] O. Ghali, O. Broux, G. Falgayrac, N. Haren, J.P. van Leeuwen, G. Penel, P. Hardouin, C. Chauveau, BMC Cell Biol. 16, 9 (2015)

[12] T. Xia, M. Kovochich, M. Liong, H. Meng, S. Kabehie, S. George, J.I. Zink, A.E. Nel, ACS Nano 3, 3273 (2009)

[13] X. Zhou, W. Feng, K. Qiu, L. Chen, W. Wang, W. Nie, X. Mo, C. He, A.C.S. Appl, Mater. Interfaces 7, 15777 (2015)

[14] J. Wang, F. Meng, W. Song, J. Jin, Q. Ma, D. Fei, L. Fang, L. Chen, Q. Wang, Y. Zhang, Int. J. Nanomed. 13, 4029 (2018)

[15] A.L. Doedens, C. Stockmann, M.P. Rubinstein, D. Liao, N. Zhang, D.G. DeNardo, L.M. Coussens, M. Karin, A.W. Goldrath, R.S. Johnson, Cancer Res. 70, 7465 (2010)

[16] J. Ikeda, T. Ichiki, H. Matsuura, E. Inoue, J. Kishimoto, A. Watanabe, C. Sankoda, S. Kitamoto, T. Tokunou, K. Takeda, G.H. Fong, K. Sunagawa, J. Am. Heart Assoc. 2, e178 (2013)

[17] Y. Qian, X. Guo, L. Che, X. Guan, B. Wu, R. Lu, M. Zhu, H. Pang, Y. Yan, Z. Ni, L. Gu, Cell Physiol. Biochem. 45, 2268 (2018) 
[18] K. Qiu, B. Chen, W. Nie, X. Zhou, W. Feng, W. Wang, L. Chen, X. Mo, Y. Wei, C. He, A.C.S. Appl, Mater. Interfaces 8, 4137 (2016)

[19] C. Liang, Y. Hu, H. Wang, D. Xia, Q. Li, J. Zhang, J. Yang, B. Li, H. Li, D. Han, M. Dong, Biomaterials 103, 170 (2016)

[20] F. Loi, L.A. Córdova, R. Zhang, J. Pajarinen, T. Lin, S.B. Goodman, Z. Yao, Stem Cell Res. Ther. 7, 15 (2016)

[21] U. Olgun, M. Gülfen, F. Üstelü, H. Arslan, Acta Metall. Sin. (Engl. Lett.) 31, 153 (2018)

[22] K. Siuzdak, M. Szkoda, J. Karczewski, J. Ryl, K. Darowicki, K. Grochowska, Acta Metall. Sin. (Engl. Lett.) 30, 1210 (2017)

[23] H. Lu, J. Wang, T. Wang, J. Zhong, Y. Bao, H. Hao, J. Nanomater. 2016, 20 (2016)

[24] R.K. Koninti, S. Palvai, S. Satpathi, S. Basu, P. Hazra, Nanoscale 8, 18436 (2016)

[25] T. Heikkilä, H.A. Santos, N. Kumar, D.Y. Murzin, J. Salonen, T. Laaksonen, L. Peltonen, J. Hirvonen, V. Lehto, Eur. J. Pharm. Biopharm. 74, 483 (2010)
[26] S. Lee, M.S. Kim, D. Lee, T.K. Kwon, D. Khang, H.S. Yun, S.H. Kim, Int. J. Nanomed. 8, 147 (2013)

[27] L. Chen, J. Liu, Y. Zhang, G. Zhang, Y. Kang, A. Chen, X. Feng, L. Shao, Nanomedicine-UK 13, 1939 (2018)

[28] J. Wang, Y. Yu, K. Lu, M. Yang, Y. Li, X. Zhou, Z. Sun, Int. J. Nanomed. 12, 809 (2017)

[29] J. Sun, X. Liu, L. Meng, W. Wei, Y. Zheng, Langmuir 30, 11002 (2014)

[30] R. Hu, C. Lin, H. Shi, H. Wang, Mater. Chem. Phys. 115, 718 (2009)

[31] X. Lu, Y. Leng, Q. Zhang, Surf. Coat. Technol. 202, 3142 (2008)

[32] W. Song, L. Zhao, K. Fang, B. Chang, Y. Zhang, J. Mater. Chem. B 3, 8567 (2015) 